\title{
Reduced prostacyclin activity in systemic lupus erythematosus
}

\author{
B. A. McVerRY, S. J. MACHIN, H. PARRY, AND A. H. Golditone \\ From the Departments of Haematology, University College Hospital, and Middlesex Hospital, London
}

SUMMARY When fresh rabbit aorta is incubated with plasma, prostacyclin, a potent inhibitor of platelet aggregation, is normally released. Plasma obtained from 2 patients with systemic lupus erythematosus (SLE) inhibited prostacyclin activity, while plasma from 22 other patients with SLE and 40 normal control subjects showed normal activity. Absence of prostacyclin activity did not appear to correlate with the clinical severity of the underlying disease. The possible association of this finding and the presence of thrombotic lesions in both patients is discussed.

The incidence of thrombotic lesions in patients with systemic lupus erythematosus (SLE) is well documented and is considerably higher than that found in the general population. ${ }^{12}$ The aetiology of these episodes is unknown.

It has recently been demonstrated that blood vessels produce prostacyclin $\left(\mathrm{PGI}_{2}\right)$, an unstable product of prostaglandin endoperoxides. ${ }^{3} \mathrm{PGI}_{2}$ is a potent inhibitor of platelet aggregation and may be responsible for the lack of adhesion and aggregation of platelets to intact vascular endothelial surfaces. ${ }^{4}$ It is possible that absent $\mathrm{PGI}_{2}$ activity may predispose to thrombotic attacks. Since plasma from SLE patients may contain antibodies directed against a large number of different antigen systems, it is of interest to determine if it may alter $\mathrm{PGI}_{2}$ activity. We present 2 patients whose plasma inhibited $\mathrm{PGI}_{2}$ activity. Both patients had a past history of vascular thrombotic episodes.

\section{Methods}

Citrated plasma was obtained from 24 patients with SLE and 40 normal control volunteers by standard techniques. The diagnosis of SLE was made in accordance with the American Rheumatism Association criteria. The age range in both groups was 20 to 50 years. Fifteen patients were taking prednisone 5-20 mg daily, 5 were taking azathioprine $100-150 \mathrm{mg}$ daily, and 5 were on no medication.

Accepted for publication 9 November 1979

Correspondence to $\mathrm{Dr}$ B. A. McVerry, Department of Haematology, Royal Liverpool Hospital, Prescot Street, Liverpool L69 3BX.
At the time of study no patient had received aspirin within 7 days, and platelet counts on all patients studied were within the normal range.

$\mathrm{PGI}_{2}$ activity was determined by a previousle described method. ${ }^{5}$ A healthy New Zealand white rabbit was killed by intravenous pentobarbitone and the aorta removed. Rings of aorta (30-60 mg wet

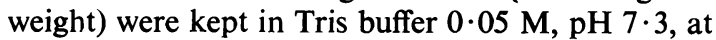
$4^{\circ} \mathrm{C}$ for up to 2 hours. $250 \mathrm{ml}$ of the patient's plasma was incubated with a ring of aorta for 5 minutes at $22^{\circ} \mathrm{C}$. The supernatant was then added to an equal volume of platelet-rich plasma (PRP) obtained from a healthy volunteer (final platelet concentration of $\left.200 \times 10^{9} / 1\right)$ for 1 minute at $22^{\circ} \mathrm{C}$. To $250 \mu$ l of this mixture, adenosine-5-diphosphate (ADP) was added to give a final ADP concentration of $2.5 \mu \mathrm{m}$, and platelet aggregation was assessed in a Payton dual channel aggregometer. Normally, inhibition of platelet aggregation by $\mathrm{PGI}_{2}$ is found. Controls consisted of the patient's plasma added to normal PRP prior to ADP aggregation but without incubation with rabbit aorta. No inhibition of platelet aggregation is normally found.

\section{Case reports}

Case 1. A 25-year-old woman was diagnosed as having SLE in 1976. Initial treatment consisted of prednisone and azathioprine. In March 1977 she developed a deep venous thrombosis in her left leg followed by a similar incident in her right leg in February 1978. An acute exacerbation of SLE in August 1978 with joint, renal, and central nervous system involvement was treated with high-dose 
prednisone and plasma exchange. At the time of the present study she was in clinical remission, though high levels of plasma DNA binding activity and circulating immune complexes were present. She was taking prednisone $15 \mathrm{mg}$ daily and azathioprine $100 \mathrm{mg}$ daily. Inhibition of $\mathrm{PGI}_{2}$ activity following incubation of the patient's plasma with rabbit aorta was demonstrated (Fig. 1). Similar inhibition was found on 2 subsequent occasions during the next 3 months.

Case 2. A 39-year-old woman with a 15-year history of SLE recently developed renal involvement with proteinuria and a reduced creatinine clearance rate. Two months before this she had an anterolateral myocardial infarction. At the time of study she was in complete remission, with normal plasma DNA binding activity and immune complex levels and was taking prednisone $10 \mathrm{mg}$ daily and azathioprine $125 \mathrm{mg}$ daily. Inhibition of $\mathrm{PGI}_{2}$ activity (similar to that of patient 1) was demonstrated. This was confirmed on a subsequent examination, but at present $\mathbf{P G I}_{2}$ activity is normal.

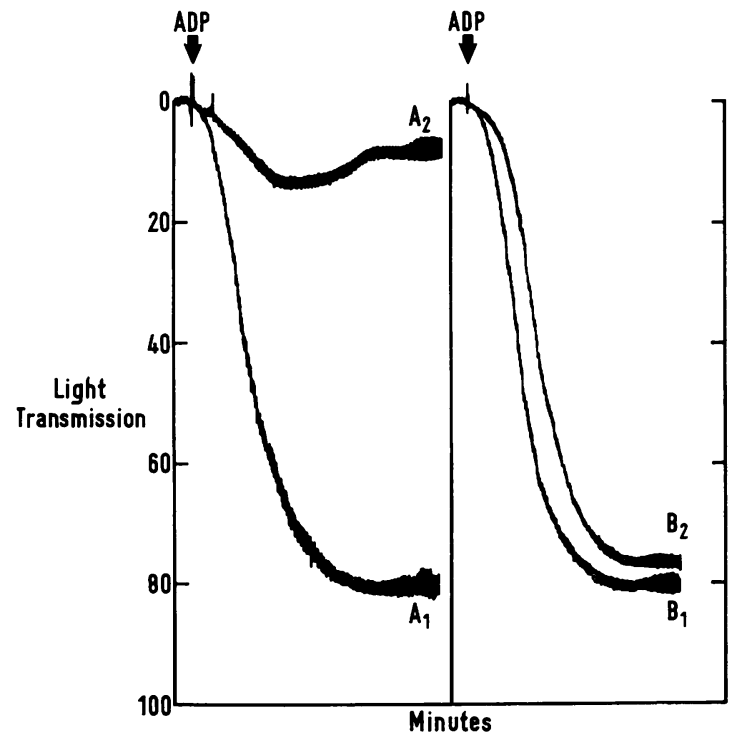

Fig. 1 ADP-induced aggregation of platelets after dilution with normal plasma $(A)$ or patient's plasma $(B)$. $A_{1}$ represents normal platelet aggregation and $A_{2}$ inhibition of aggregation by added prostacyclin. Prostacyclin did not inhibit aggregation in the presence of the patient's plasma $\left(B_{2}\right)$.

\section{Discussion}

Prostacyclin is produced by vascular tissue and is a potent vasodilator and inhibitor of platelet aggregation. It has been demonstrated that $\mathrm{PGI}_{2}$ prevents platelets adhering to normal vascular endothelial surfaces ${ }^{4}$ in an in-vitro system. The role of $\mathrm{PGI}_{2}$ in the prevention of arterial and venous thrombotic disease is at present unknown. It had been suggested that diseases such as thrombotic thrombocytopenic purpura lack $\mathrm{PGI}_{2}$ owing to a deficiency of a necessary plasma factor. ${ }^{6} \mathbf{P G I}_{2}$ has been shown to significantly improve the symptoms of patients with severe, advanced peripheral vascular disease possibly owing to its ability to disperse platelet aggregates. ${ }^{7}$

It is therefore likely that any disease which inhibits the formation of $\mathrm{PGI}_{2}$ or alters the activity of released $\mathrm{PGI}_{2}$ renders a patient more susceptible to thrombotic episodes. The plasma from 2 of our 24 patients with SLE clearly impaired the activity of $\mathrm{PGI}_{2}$ obtained from rabbit aorta. Furthermore, these were the only patients in this study group to have a previous history of thrombotic episodes. While a cause-and-effect relationship cannot be determined on such a small number of patients, the finding is nevertheless interesting and worthy of further study.

While inhibitory activity is no longer detectable in both patients' plasma, it must be recognised that our test system is crude and lacks sensitivity. More sensitive test systems may demonstrate a higher incidence of absent plasma $\mathrm{PGI}_{2}$ activity in this group of patients.

\section{References}

1 Dubois E L. Lupus Erythematosus, 2nd ed. University of Southern California Press, 1976.

2 Hamilton C R, Tumulty P A. Thrombosis of renal veins and inferior vena cava complicating lupus nephritis. JAMA 1968; 206: 2315-6.

3 Moncada S, Gryglewski R, Bunting S, Vane J R. An enzyme isolated from arteries transforms prostaglandin endoperoxides to an unstable substance that inhibits platelet aggregation. Nature 1976; 263: 663-5.

4 Weiss $\mathrm{H} \mathrm{J}$, Turitto V T. Prostacyclin (prostaglandin $\mathbf{I}_{2}$ and $\mathrm{PGI}_{2}$ ) inhibits platelet adhesion and thrombus formation on subendothelium. Blood 1979; 53: 244-50.

5 Moncada S, Higgs E A, Vane J R. Human arterial and venous tissues generate prostacyclin (prostaglandin $\mathrm{X})$, a potent inhibitor of platelet aggregation. Lancet 1977; 1: 18-20.

6 Remuzzi G, Misiani R, Marchesi D, et al. Haemolyticuraemic syndrome; deficiency of plasma factor(s) regulating prostacyclin activity? Lancet $1978 ; 2$ : 871-2.

7 Szczeklik A, Gryglewski R, Nikankowski R, Szczeklik G, Skawinski S, Musiak G. Anti-platelet and vasodilatory action of prostacyclin $\left(\mathrm{PGI}_{2}\right)$ in healthy men and in patients with peripheral artery disease. Thromb Haemostas 1979; 42: 161 (abstract). 